\title{
FOURIER DECOMPOSITIONS WITH POSITIVE COEFFICIENTS IN COMPACT GELFAND PAIRS
}

\author{
BRIAN E. BLANK
}

(Communicated by J. Marshall Ash)

\begin{abstract}
For $G$ a compact separable Hausdorff topological group and for $1<p \leq 2$ the finiteness of the Hausdorff-Young sequence operator is established for functions in $L^{1}(G)$ with positive Fourier decompositions and which are $p$ th-power integrable in a neighborhood of the identity. A similar result is established in the context of compact Gelfand pairs.
\end{abstract}

In the early 1950s Norbert Wiener proved but did not publish the result that an integrable function on the circle group $G=\mathbb{R} / 2 \pi \mathbb{Z}$ that is in $L^{2}(U)$ for a neighborhood $U$ of the identity element and that has nonnegative Fourier coefficients is in $L^{2}(G)$ [2, pp. 242, 250; 7]. When $L^{2}$ is replaced by $L^{p}$ in both occurrences in the statement of the theorem, Wainger [8] (for $1<p<2$ ) and Shapiro [7] (for $p \in(2, \infty)-2 \mathbb{Z}^{+}$) have given counterexamples. Remarkably, the correct formulation of a generalization of Wiener's theorem did not appear until 1987 when Ash, Rains, and Vagi [1] proved the following (for $G=\mathbb{R} / 2 \pi \mathbb{Z}$, $\widehat{G}=\mathbb{Z}$, and normalized Haar measure $\nu$ on $G$ given by $(2 \pi)^{-1}$ Lebesgue measure and Plancherel measure $\hat{\nu}$ on $\mathbb{Z}$ given by $(2 \pi)^{-1}$ counting measure; of course, property (iv) is no restriction in this abelian group setting):

Theorem A. If

(i) $f \in L^{1}(G, d \nu)$,

(ii) $\left.f\right|_{U} \in L^{p}\left(U,\left.d \nu\right|_{U}\right)(1<p \leq 2)$ for a neighborhood $U$ of the identity element $e$ of $G$,

(iii) $\hat{f}(\alpha) \geq 0 \quad(\alpha \in \widehat{G})$, and

(iv) $f(x y)=f(y x) \quad(x, y \in G)$,

then $\hat{f} \in L^{q}(\widehat{G}, d \hat{\nu})$ where $q=p /(p-1)$.

Even more striking in view of other possible proofs and the history, is the elegance and simplicity of their argument. Subsequently, Kawazoe and Miyazaki, using the method in [1], proved Theorem A for compact semisimple Lie groups $G$ and an analogous result for spherical Fourier decompositions of $K$-bi-invariant functions on compact symmetric spaces (see Theorem B) [6]. It is the aim of this note to observe that by using the Peter-Weyl theory, the proofs in [6] apply for arbitrary separable compact Hausdorff topological groups in the case

Received by the editors March 25, 1991.

1991 Mathematics Subject Classification. Primary 43A30, 43A15. 
of Theorem A and compact Gelfand pairs in the case of Theorem B. While the gain in generality is not great, we feel, the gain in lucidity by dropping the differential structure (the Lie hypothesis) and the algebraic structure (the semisimple hypothesis) is worthwhile, particularly in distinguishing the nature of these results from more subtle phenomena such as multiplier theorems where the make-up of $\widehat{G}$ is important. We emphasize that the proofs of Kawazoe and Miyazaki need not be changed for this added generality. In what follows, all facts without specific citations may be found in the standard references [4, 5].

Let $G$ be a separable compact Hausdorff topological group, and let $\widehat{G}$ denote the set of equivalence classes of irreducible unitary representations of $G$. Convolution is defined by $\varphi * \psi(x)=\int_{G} \varphi\left(x y^{-1}\right) \psi(y) d \nu(y)$ where $\nu$ is a normalized bi-invariant measure on $G$. For $\alpha$ in $\widehat{G}$, let $d_{\alpha}=\operatorname{dim}_{\mathbb{C}} H_{\pi}$ and $\chi_{\alpha}=\operatorname{Tr} \pi$ where $\left(\pi, H_{\pi}\right) \in \alpha$. Let $\hat{\nu}$ be the measure on the power set of $\widehat{G}$ given by $\hat{\nu}(\{\alpha\})=d_{\alpha}^{2}$. Basic to the Peter-Weyl theory is that $d_{\alpha}<\infty$ for every $\alpha \in \widehat{G}$. For $f$ in $L^{1}(G)$ and $\left(\pi, H_{\pi}\right) \in \alpha \in \widehat{G}$, let $\mathscr{F} f(\pi)=\int_{G} f(x) \pi\left(x^{-1}\right) d \nu(x)$. The Hilbert-Schmidt norm of $\hat{f}(\pi)$ depends only on $\alpha$ and may be computed by $\|\mathscr{F} f(\alpha)\|_{\mathrm{HS}}^{2}=\chi_{\alpha} * f * \check{f}(e)$ where $\check{f}(x)=\overline{f\left(x^{-1}\right)}$. Other aspects of the Peter-Weyl theory are the Plancherel and Fourier inversion formulas:

$$
\begin{aligned}
\|f\|_{L^{2}(G)}^{2} & =\sum_{\alpha \in \widehat{G}} d_{\alpha}\|\mathscr{F} f(\alpha)\|_{\mathrm{HS}}^{2} \quad\left(f \in L^{2}(G)\right), \\
f & =\sum_{\alpha \in \widehat{G}} d_{\alpha} \chi_{\alpha} * f \quad\left(f \in L^{2}(G)\right) .
\end{aligned}
$$

Furthermore, $\left\{\chi_{\alpha} \mid \alpha \in \widehat{G}\right\}$ is an orthonormal basis for the class (or central) functions in $L^{2}(G)$ : if $L_{\mathscr{G}}^{p}(G)=\left\{f \in L^{p}(G) \mid f\left(\xi x \xi^{-1}\right)=f(x)\right.$ for every $\xi$ for $\nu$-a.e. $x\}$ and if $\hat{f}(\alpha)=d_{\alpha}^{-1}\left\langle f, \chi_{\alpha}\right\rangle$ then (1) and (2) simplify to

$$
\begin{gathered}
\|f\|_{L^{2}(G)}^{2}=\sum_{\alpha \in \widehat{G}} d_{\alpha}^{2}|\hat{f}(\alpha)|^{2}=\|\hat{f}\|_{L^{2}(\widehat{G})}^{2} \quad\left(f \in L_{\mathscr{G}}^{2}(G)\right), \\
f=\sum_{\alpha \in \widehat{G}} d_{\alpha} \hat{f}(\alpha) \chi_{\alpha} \quad\left(f \in L_{\mathscr{G}}^{2}(G)\right) .
\end{gathered}
$$

By the standard Riesz-Thorin interpolation argument based on (3) and the trivial estimate $\|\hat{f}\|_{L^{\infty}(G)} \leq\|f\|_{L^{1}(G)}$, we have the Hausdorff-Young inequality

$$
\|\hat{f}\|_{L^{q}(\widehat{G})} \leq\|f\|_{L^{p}(G)} \quad\left(f \in L_{\mathscr{G}}^{p}(G), 1 \leq p \leq 2, q=p /(p-1)\right) .
$$

Lemma. Let $U$ be a neighborhood of $e$ in $G$. There exists a continuous function $h$ on $G$ with support in $U$ such that $\hat{h} \geq 0$ and $\hat{h}\left(\tau_{G}\right)=\int_{G} h d \nu=1$ where $\tau_{G}$ is the trivial representation of $G$. Furthermore, $h$ may be chosen to be central.

Proof. The required function $h$ is constructed in a manner analogous to that of [6, Lemma 4.2] without using the differential structure assumed there. Let $W$ be a symmetric neighborhood of the identity such that $W^{2} \subset U$. Let $g=\nu(W)^{-1} \chi_{W}$. Then $h_{0}=g * g$ is continuous with support in $U$ and $\int_{G} h_{0} d \nu=1$. Since $g=\breve{g}$, it follows that $h_{0}$ is positive definite and, therefore, $\hat{h}_{0} \geq 0$. Then $h(x)=\int_{G} h_{0}\left(y x y^{-1}\right) d \nu(y)$ is central and shares the previously noted properties of $h_{0}$. 
By the complete reducibility of finite-dimensional unitary representations, we see that for $\alpha, \beta, \gamma \in \widehat{G}$ there are nonnegative integers $m(\alpha, \beta: \gamma)$ such that

$$
\chi_{\alpha} \chi_{\beta}=\sum_{\gamma \in \widehat{G}} m(\alpha, \beta: \gamma) \chi_{\gamma} \quad(m(\alpha, \beta: \gamma) \geq 0) .
$$

The proof of Theorem $A$ is now immediate. Computation using (4) yields

$$
(h \cdot f)^{\wedge}(\gamma)=d_{\gamma}^{-1} \sum_{\alpha, \beta \in \widehat{G}} d_{\alpha} d_{\beta} m(\alpha, \beta: \gamma) \cdot \hat{h}(\alpha) \cdot \hat{f}(\beta) \quad\left(f \in L_{\mathscr{C}}^{1}(G)\right) .
$$

Since $m\left(\tau_{G}, \gamma: \gamma\right)=1$ and since the constituents of the sum above are all nonnegative, we have $\hat{f}(\gamma) \leq(h \cdot f)^{\wedge}(\gamma) \quad(\gamma \in \widehat{G})$. Since $h \cdot f \in L^{p}(G)$, Theorem A now follows from (5).

We remark that the restriction to the class of central functions was mainly for ease of notation. For general $f$ we must replace $\chi_{\alpha}$ with an orthogonal basis $\left\{\varphi_{\alpha, i}: 1 \leq i \leq d_{\alpha}^{2}\right\}$ of the $\alpha$-isotypical subspace of $L^{2}(G)$ where $\left\{\varphi_{\alpha, i}\right\}$ represents an enumeration of the matrix coefficients $\left\{x \rightarrow\left\langle\pi(x) u_{j}, u_{k}\right\rangle_{H_{\pi}}\right\}$ for an orthonormal basis $\left\{u_{j}\right\}$ of $H_{\pi}\left(\left(\pi, H_{\pi}\right) \in \alpha\right)$. We then get a formula analogous to (6) for these functions; it is this type of formula which is necessary for these types of theorems and which seems to be special to orthogonal decompositions that are group Fourier series. By Schur orthogonality, $\left\{d_{\alpha}^{1 / 2} \varphi_{\alpha, i}\right\}$ are orthonormal. Let $\hat{f}(\alpha, i)=\left\langle f, \varphi_{\alpha, i}\right\rangle$ for $f$ in $L^{2}(G)$. Using (1) and $\left\|\varphi_{n}\right\|_{\infty}=1$, we get $\sum_{\alpha} \sum_{i} d_{\alpha}|\hat{f}(\alpha, i)|^{q} \leq\|f\|_{L^{p}(G)}$. If in Theorem A we use $\bigcup_{\alpha \in \widehat{G}}\left\{(\alpha, i) \mid 1 \leq i \leq d_{\alpha}^{2}\right\}$ instead of $\widehat{G}$ and set $\hat{\nu}(\{(\alpha, i)\})=d_{\alpha}$, then Theorem A remains true without hypothesis (iv).

Now suppose that $X=(G, K)$ is a compact Gelfand pair: $G$ is as above and $K$ is a closed subgroup such that the continuous $K$-bi-invariant functions $C(G / / K)$ form a commutative convolution algebra. Let $\widehat{G}_{K}$ denote the subset of $\widehat{G}$ consisting of representations with a $K$-fixed vector. Let $\nu$ and $\nu_{K}$ denote normalized Haar measure on $G$ and $K$ respectively. For $\alpha \in \widehat{G}_{K}$ let $\varphi_{\alpha}(x)=\int_{K} \chi_{\alpha}(x k) d \nu_{K}(k)$. Let $L^{p}(G / / K)$ denote the space of $K$-bi-invariant functions in $L^{p}(G, d \nu)$. Now let $\hat{\nu}_{X}$ denote the measure on $\widehat{G}_{K}$ given by $\hat{\nu}_{X}(\{\alpha\})=d_{\alpha} \quad\left(\alpha \in \widehat{G}_{K}\right)$; this is, in effect, the Plancherel-Godement measure on $\widehat{G}_{K}$ (and, in particular, $\hat{\nu}_{X}$ is not the restriction of $\hat{\nu}$ to the power set of $\left.\widehat{G}_{K}\right)$. The correspondence $\alpha \rightarrow \varphi_{\alpha}$ is a bijection between $\widehat{G}_{K}$ and the set of positive-definite spherical functions on $G$. For $f \in L^{1}(G / / K)$, let $\hat{f}(\alpha)=\left\langle f, \chi_{\alpha}\right\rangle_{L^{2}(G)}=\left\langle f, \varphi_{\alpha}\right\rangle_{L^{2}(G)}$. By either the Peter-Weyl theory (including the functional equation of spherical functions) or the Bochner-Godement theory [3], one has

$$
\begin{gathered}
\|f\|_{L^{2}(G)}^{2}=\sum_{\alpha \in \widehat{G}_{K}} d_{\alpha}|\hat{f}(\alpha)|^{2}=\|\hat{f}\|_{L^{2}\left(\widehat{G}_{K}\right)}^{2} \quad\left(f \in L^{2}(G / / K)\right), \\
f=\sum_{\alpha \in \widehat{G}_{K}} d_{\alpha} \hat{f}(\alpha) \varphi_{\alpha} \quad\left(f \in L^{2}(G / / K)\right) .
\end{gathered}
$$

Since $\varphi_{\alpha}$ is positive-definite and spherical, we have $\left\|\varphi_{\alpha}\right\|_{\infty} \leq \varphi_{\alpha}(e)=1$ which 
implies that $\|\hat{f}\|_{L^{\infty}\left(\widehat{G}_{K}\right)} \leq\|f\|_{L^{1}(G)}$. Then Riesz-Thorin interpolation gives

$$
\|\hat{f}\|_{L^{q}\left(\widehat{G}_{K}\right)} \leq\|f\|_{L^{p}(G)} \quad\left(f \in L^{p}(G / / K), 1 \leq p \leq 2, q=p /(p-1)\right) .
$$

Again crucial to a generalization of the Ash-Rains-Vagi theorem to a noncommutative setting is that

$$
\varphi_{\alpha}(x) \cdot \varphi_{\beta}(x)=\sum_{\gamma \in \widehat{G}_{K}} d_{\gamma} c(\alpha, \beta: \gamma) \varphi_{\gamma}(x), \quad c(\alpha, \beta: \gamma) \geq 0\left(\alpha, \beta \in \widehat{G}_{K}\right) .
$$

Once again, the proof given in [6, Lemmas 3.2, 3.3] is valid in greater generality. That proof, however, is based on an argument given by Flensted-Jensen and Koornwinder for noncompact symmetric spaces. Here it is more natural, perhaps, to observe that since $G$ is compact, $\varphi_{\alpha}$ and $\varphi_{\beta}$ are positive-definite and, therefore, so is their product $[5, \S 32.9]$, from which (10) is immediate. Finally we need the auxiliary function $h$. Shrink the set $W$ of the previous lemma so that $K W K \subset U$, let $h_{0}$ be the function in the lemma associated with this new $W$, and set $h(x)=\int_{K \times K} h_{0}\left(k_{1} x k_{2}\right) d \nu_{K}\left(k_{1}\right) d \nu_{K}\left(k_{2}\right)$. Then $\hat{h} \geq 0$ on $\widehat{G}_{K}, \hat{h}\left(\tau_{G}\right)=1, h$ is supported in $U$, and $h \in C(G / / K)$. Now, by direct computation

$$
(h \cdot f)(\gamma)=\sum_{\alpha, \beta \in \widehat{G}_{K}} d_{\alpha} d_{\beta} c(\alpha, \beta: \gamma) \hat{h}(\alpha) \cdot \hat{f}(\beta) \quad\left(f \in L^{1}(G / / K)\right) .
$$

Theorem B. Let $(G, K)$ be a compact Gelfand pair. Suppose that $f \in L^{1}(G / / K)$ and that $\left.f\right|_{U} \in L^{p}\left(U,\left.d \nu\right|_{U}\right)$ for some neighborhood $U$ of $e$ in $G$ and $1 \leq p \leq$ 2. If $\hat{f}(\gamma) \geq 0$ for all $\gamma$ in $\widehat{G}_{K}$ then $\hat{f}$ is in $L^{q}\left(\widehat{G}_{K}, d \hat{\nu}_{X}\right)$ where $q=p /(p-1)$. Proof. Since $c\left(\tau_{G}, \gamma: \gamma\right)=d_{\gamma}^{-1}$, it follows from (10) and (11) that $\hat{f}(\gamma) \leq$ $(h \cdot f)^{\wedge}(\gamma) \quad\left(\gamma \in \widehat{G}_{K}\right)$. The result now follows from Hausdorff-Young applied to $h \cdot f$ which is in $L^{p}(G / / K)$ by construction.

\section{REFERENCES}

1. J. Marshall Ash, Michael Rains, and Stephen Vagi, Fourier series with positive coefficients, Proc. Amer. Math. Soc. 101 (1987), 392-393.

2. Ralph Philip Boas, Jr., Entire functions, Academic Press, New York, 1954.

3. R. Godement, A theory of spherical functions. I, Trans. Amer. Math. Soc. 73 (1952), 496-556.

4. Sigurdur Helgason, Groups and geometric analysis, Academic Press, Orlando, FL, 1984.

5. Edwin Hewitt and Kenneth A. Ross, Abstract harmonic analysis, Vol. I, II, Springer-Verlag, Berlin, 1963, 1970.

6. Takeshi Kawazoe and Hiroshi Miyazaki, Fourier series with nonnegative coefficients on compact semisimple Lie groups, Tokyo J. Math. 12 (1989), 241-246.

7. Harold S. Shapiro, Majorant problems for Fourier coefficients, Quart. J. Math. Oxford Ser. (2) 26 (1975), 9-18.

8. S. Wainger, A problem of Wiener and the failure of a principle for Fourier series with positive coefficients, Proc. Amer. Math. Soc. 20 (1969), 16-18.

Department of Mathematics, Washington University, St. Louis, Missouri 63130

E-mail address: c31801bb@wuvmd 